\title{
Patient waiting time and satisfaction at a Tertiary Dental School
}

SADJ July 2018, Vol 73 no 6 p400 - p405

PD Motloba ${ }^{1}$, O Ncube ${ }^{2}$, LN Makwakwa ${ }^{3}$, ML Machete. $^{4}$

\section{ABSTRACT}

Introduction: The goal of health care systems is to provide timeous, high quality, equitable and efficient services to patients. Exceptionally long waiting times remain worldwide the primary concern for patients, ahead of service quality attributes such as behaviour and attitudes of staff, communication and service environment. ${ }^{1}$ The intention of the patient to revisit or even to refer family to the institution is profoundly influenced by waiting times. ${ }^{2}$

Aim and objectives: To measure the association between satisfaction and patient waiting times, and intention to revisit or to refer others to the Medunsa Oral Health Centre $(\mathrm{MOHC})$.

Methods: A descriptive cross-sectional survey was undertaken over a three month period (August to October) and 149 consenting participants completed a questionnaire. Data on waiting times were collected at every hospital section visited each day.

Results: Mean waiting time for consultations for the 149 participants was 84.84 minutes. Satisfaction over waiting time, state of the Hospital and quality of care ranged between $60 \%-80 \%$. Perceptions about waiting time, justification of waiting time, intention to revisit and to refer others were significantly associated with patient satisfaction.

Conclusion: Patients who perceive their waiting times to be long are unlikely to revisit or refer family or friends to the facility in future.

1. Pagollang D Motloba: BDS(Medunsa), MPH(Epidemiology) (Tulane), M Dent(Comm Dent)(Medunsa), MBL(Unisa). Chief Specialist, Head of Department Community Dentistry *

2. Ozias Ncube: BSc (Hons), MSc. Senior Lecturer, Department of Supply Chain Management at UNISA GSBL *

3. Nokukhanya L Makwakwa: BDT, BDS (Medunsa) Postgraduate Dip in Clinical Dentistry (UWC), MPH (UL). Registrar Community Dentistry *

4. Motimedi L Machete: BDS (UL), MDS (SMU). Lecturer, Community Dentistry *

*School of Oral Health Sciences - Sefako Makgatho Health Science University (SMU), Department of Community Dentistry.

Corresponding author

Pagollang D Motloba:

HOD, Department Community Dentistry, Sefako Makgatho Health Science

University. Tel: +27 12521 4848. E-mail: pagollang.motloba@smu.ac.za

\section{INTRODUCTION}

The goal of a health system is to provide quality care and services timeously to all patients. ${ }^{1}$ Consequently, health providers invest in systems and activities aimed at providing quality healthcare services to encourage customer satisfaction. Patients who are satisfied with the service they receive are more inclined to return for further appointments or even to refer others to the same practitioner or facility. ${ }^{3}$ Despite great innovation and investment in health systems, patient satisfaction remains a major challenge for health care providers, especially nowadays in the era when health care services are regarded as a commodity. ${ }^{4,5}$

Satisfaction is an emotional state, arrived at because the customer has evaluated the service or product received against their expectations. ${ }^{6,7}$ Healthcare managers ought to recognize patient satisfaction as a necessary and appropriate outcome of the system and that it is an appropriate measure of successful service provision. Patient satisfaction is even more important in relation to the determinants and performance of health services. ${ }^{8,9}$

Several factors are thought to impact patient satisfaction and related health outcomes.9,10 Excessive waiting time to see a doctor or health professional is a frequently cited reason why patients are dissatisfied with healthcare services. ${ }^{10}$

The problem of waiting time in healthcare institutions has been unequivocally ranked the top concern by patients, specifically in the outpatient department, ahead of staff problems, communication, behaviour and attitude.11,12 Waiting times for patients presenting at emergency facilities are reported to be the worst compared with those experienced in general care and cold medical cases..$^{13}$ Long waiting times threaten access to healthcare and impact on the quality of care provided. Ideally, healthcare should be provided when needed, and without undue delay.

Nowadays, private sector healthcare services are a traded commodity, such that protracted and long waiting times will seriously affect the number of clients processed, and ultimately impact on profit and related bottom lines. ${ }^{14}$ Furthermore, waiting times negatively affect the clients' perception, and hence the reputation, of the facility or 
practice and ultimately result in a decreased likelihood of the patients referring others through word of mouth.

For public health institutions such as SMU, which have budgetary constraints, as well as targets set by the provincial department, efficiency is a key performance area. These performance indicators include headcounts, satisfied patients and certain specified waiting times. It is hence mandatory for teaching hospitals to be efficient in the use of resources in order to achieve these national and provincial core standards of care.

Dental teaching institutions have not been extensively studied with respect to this phenomenon yet they post longer waiting times compared with other health facilities. The rigorous treatment protocols and guidelines followed in teaching institutions may be structured to minimise risks, yet they are lengthy, deliberate and often result in an increased consultation time per patient. When student training is taken into account the waiting times extend even further.

This study was conducted to evaluate the waiting times experienced in the emergency departments at a dental teaching hospital in South Africa. This study further sought to determine the association between waiting times and patient satisfaction. Information from this study is likely to be used by managers, doctors, students and other health personnel to improve on waiting times, patient satisfaction and ultimately on the quality of care provided.

\section{METHODOLOGY}

\section{Study design and sampling}

The study was conducted at Medunsa Oral Health Centre $(\mathrm{MOHC})$, a tertiary teaching oral and dental hospital that forms part of Sefako Makgatho Health Sciences University. This centre is primarily responsible for rendering oral health services to the public, as well as the teaching and training of undergraduate and postgraduate dental students. The facility caters for oral and dental needs of patients from surrounding clinics and hospitals and serves as a referral centre for hospitals from neighbouring provinces North West, Mpumalanga and Limpopo.

A survey was conducted over a period of three months on patients who attended the Maxillo-facial and Oral Surgery department (MFOS) and Emergency department of the dental hospital. These two clinics were chosen because they represent the bulk of emergency relief of pain and sepsis clinical work conducted at the facility. All patients who attended MFOS and Emergency had been examined at the Diagnostics department and referred according to their specific individual needs. These three departments are considered as the gateway to the hospital services, and it is here that the patient gains first impressions of the centre. This exposure often influences the patient's acceptance of the hospital and hence it is essential to ensure that services in these departments provide a good experience for those attending.

Based on the patient load in the outpatients departments, and relying on a margin of error of $5 \%$ and a $95 \%$ confidence interval, the required sample size was estimated at 122 participants. Systematic random sampling was used to select every fifth patient receiving care at MFOS and Emergency unit until the required sample size was reached. To be eligible to participate in the study, the individual was required to be receiving treatment on the day of survey, to be of consenting age and capable of making autonomous decisions about treatment.

A previously validated questionnaire ${ }^{15}$ was used to collect data and was administered by three calibrated researchers. The researchers remained available to the study participants to provide any required assistance. The first part of the questionnaire contained questions relating to socio-demographic data and waiting time during the visit. The second part was designed to measure the patients' perception, and views about the delivery of service.

Ethical clearance was granted by the Unisa School of Business Science (UNISA-SBL), and the Management of Medunsa Oral Health Centre granted permission for the study to be undertaken. Participation in the study was voluntary and respondents were informed they could withdraw at any time with no consequences.

\section{Socio-demographic details}

Each study participant was requested to provide information about his or her age, sex and socio-economic status. Socio-economic status was measured according to annual individual income or net family income including assets. Participants who indicated an individual income of less than R35 000 per annum or a net family income of less than R75 000 per annum were classified as having a low socio-economic status. Participants who indicated an individual income of more than R35 000 or a family income more than R75 000 were classified as having a medium to high socio-economic status.

\section{Reason for visiting the hospital}

Participants were asked to indicate how they came to attend the hospital (whether self-referred or referred by a third party). In addition, participants were asked to indicate the frequency of visits they had made to the hospital (whether first visit, second visit or more) and to report in which department they were currently receiving treatment (whether Emergency or Maxillofacial Surgery).

\section{DATA COLLECTION}

\section{Assessment of patient waiting time}

Details of every patient entering $\mathrm{MOHC}$ are routinely captured by the security personnel for the collection of statistics on daily visits. This study utilised the recording of time at several stages during the period the patients spent in the hospital Time of entry $\left(T_{1}\right)$, is recorded on arrival. Patients then await their turn with the administration clerks and on that contact $T_{2}$ is recorded in the administration sheet for the day. Patients are then released to wait at the reception area for a dental consultation. and $T_{3}$ is recorded by dental assistants when patients are admitted to the Diagnostics Department. Patients are seen by dentists at this section, and $T_{4}$ is recorded when the consultation is completed and the patient is referred for treatment at MFOS or the Emergency Department (Careline). $T_{5}$ and $T_{6}$ are recorded respectively when patients enter and leave these treatment sections. On completion of treatment, all patients are signed out by the security personnel when they exit $\mathrm{MOHC}$, and $\mathrm{T}_{7}$ is recorded (Figure 1.). 


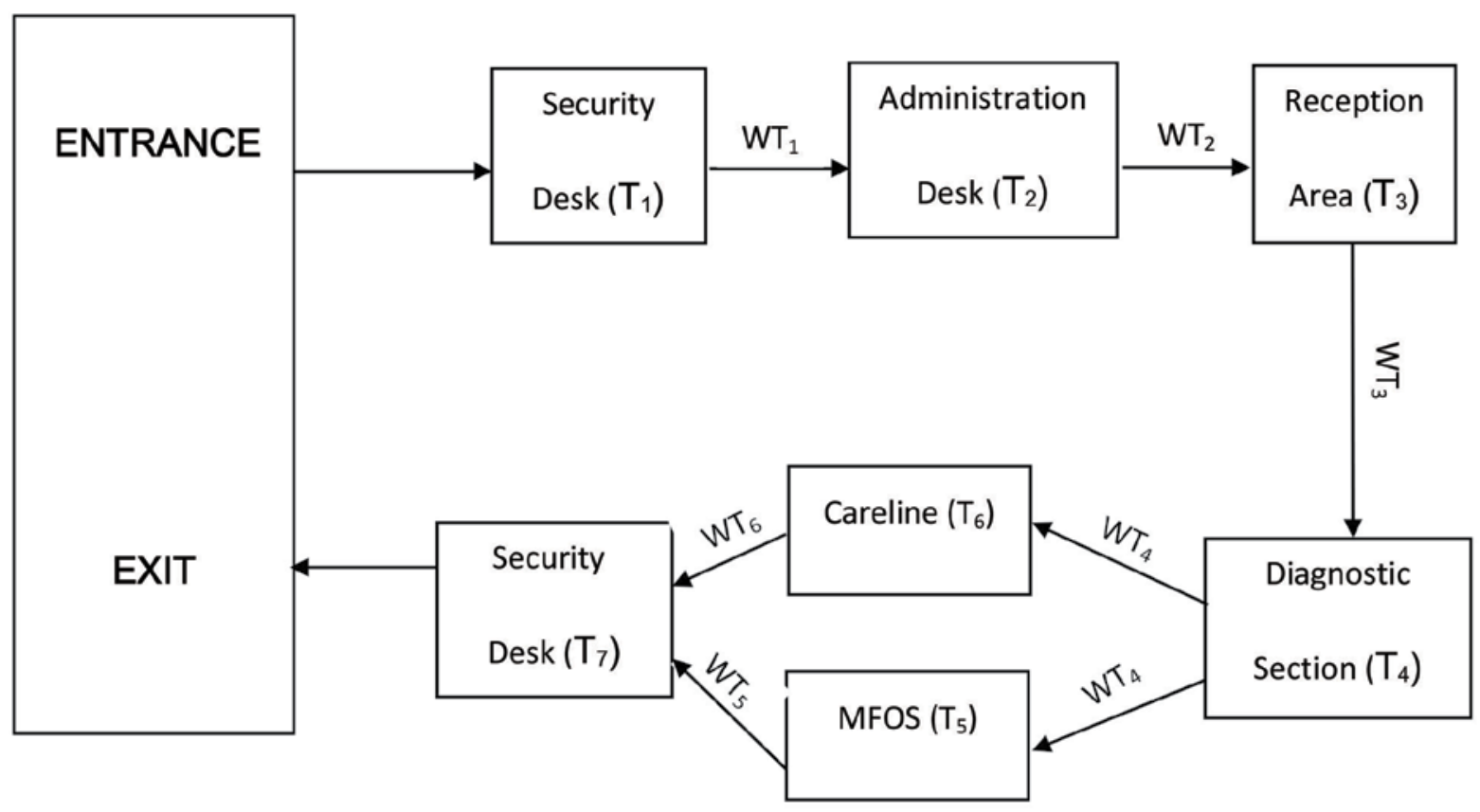

Figure 1: Patient flow and recording of waiting time.

Based on these recoded data, the following waiting times were computed: - Admin waiting time, $\mathrm{WT}_{1}\left(\mathrm{~T}_{2}-\mathrm{T}_{1}\right)$ being the time elapsed from when the patient entered $\mathrm{MOHC}$ to the time he/she was attended by the administration personnel. Reception Wait, $\mathrm{WT}_{2}\left(\mathrm{~T}_{3}-\mathrm{T}_{2}\right)$, the time spent in reception area, before seeing the dentist. Diagnosis Wait, $\mathrm{WT}_{3}\left(\mathrm{~T}_{4}-\mathrm{T}_{3}\right)$, the period spent waiting in the Diagnostic Department before being seen by a dentist. Treatment Wait, $\mathrm{WT}_{4}\left(\mathrm{~T}_{5}-\mathrm{T}_{4}\right)$ the time during which the patient awaited specific treatment at MFOS or Careline, and $\mathrm{WT}_{6}\left(\mathrm{~T}_{6}-\mathrm{T}_{5}\right)$, indicates the time spent actually receiving treatment at MFOS and Careline respectively. Total waiting time at $\mathrm{MOHC}$ was computed as $\mathrm{T}_{7}-\mathrm{T}_{1}$ (Figure 1).

Participants' perceptions about waiting time This study used the dimensions defined by the service quality (SERVQUAL) model, which has been adopted by other studies that explored the perceptions of patients of the quality of various services..$^{15}$ Questions were adapted from this model to produce direct and indirect closeended questions. Participants were asked to indicate their perceptions, thoughts and feelings about the waiting time spent in the hospital. The participants were requested to indicate whether they thought the waiting time was "long", "adequate", or "short". In addition, participants had to indicate how they felt about the waiting time - whether they were "satisfied", "dissatisfied" or "very dissatisfied". In addition, participants were asked to indicate what were their perceptions regarding the causes of long waiting times which may have been experienced in the overall time spent at the dental hospital. Response options included "Long search for patient files", "Lost files", "Large number of patients", "Doctors arrive late" and "Doctors spend too much time with patients".

Participants' views about care and service received Participants were asked to indicate their views about service attributes such as waiting times, state of the hospital, attitude of the attending dentists, and quality of care received. Standard answers were provided and the participants were required to indicate "yes", "no" or "not sure" on each of the items.

\section{Statistical Analysis}

Statistical data analysis was computed using SPSS version 23, and included measures of central tendency and dispersion. The frequency proportions were collated as a univariate analysis. Inferential statistics, namely t-test and chi-square tests were applied where appropriate to investigate bivariate relationships. Logistic regression analysis was done to determine the influence of factors possibly affecting overall satisfaction. All tests were considered significant at $p<0.05$.

\section{RESULTS}

A total of 149 participants were enrolled in the study. The median age of the participants was 30 years, with a mean of 33,16 and standard deviation of 16.02 years. The greater proportion (55.7\%) of the participants were between 21 and 40 years of age. Other characteristics of the participants are presented on Table 1. Most of the participants were female (62.4\%) and mainly hailed from low socioeconomic backgrounds (78.5\%).

\begin{tabular}{llc}
\hline \multicolumn{2}{l}{ Table 1: Descriptive Characteristics of Participants } \\
Variable & & $\begin{array}{c}\text { Percent (Frequency) } \\
\text { N=149 }\end{array}$ \\
\hline \multirow{2}{*}{ Gender } & Female & $62.4(93)$ \\
& Male & $37.6(56)$ \\
SES (Income) & Low & $78.5(117)$ \\
& Medium to High & $21.5(32)$ \\
\hline \multirow{2}{*}{ Referral } & Self & $83.9(125)$ \\
& Third Party & $16.1(24)$ \\
Clinic Visited & MFOS & $69.1(103)$ \\
& Emergency & $30.9(46)$ \\
Frequency of & Once & $60.4(90)$ \\
Visits & Twice or More & $39.6(59)$ \\
\hline
\end{tabular}


Details of the waiting times and time spent in the various Departments are shown on Table 2. The average waiting time before being seen at Diagnostics was 84.84 minutes. Of the time spent in consultation, the examinations accounted for the shortest time, 45.84 minutes as opposed to 52.75 and 80.38 minutes for MFOS and Emergency Departments respectively. The mean total cumulative time spent with doctors during treatment was approximately 110.94 minutes. Overall, it takes an average of four (4) hours for patients to interact with the system and to complete any form of emergency treatment at $\mathrm{MOHC}$ on any given day.

The data in Table 3 seem to indicate that most of the participants (40$64 \%)$ thought the waiting time was either as expected or short. Furthermore, approximately $80 \%$ of the participants were satisfied with the length of waiting time. Table 4 shows the reasons perceived by the participants for long waiting times. These included late arrival of doctors, doctors taking a long time to treat patients, the large volume of patients and protracted time waiting to receive a file.

According to Table 5, participants generally indicated that they were satisfied with the waiting times and care received at the hospital. A high percentage of participants indicated that the waiting time they experienced was justified. Similarly, the majority of the participants were satisfied with the state of the hospital and the care received at the hospital. An overwhelming majority (85.9\%) of participants indicated their intention to visit the hospital again in the future as well as a willingness to refer friends and family to $\mathrm{MOHC}$ in the future $(81.9 \%)$.

The logistic regression analysis of factors associated with patient satisfaction is shown on Table 6. These results suggest that an increase in the waiting time prior to being attended to by the dentist and an increased length of time spent with the dentist was associated with a decrease in the satisfaction reported by the participants. Surprisingly however, patients who spent more time in the hospital were in general more likely to be satisfied than those who spent less time.

\section{DISCUSSION}

The mean age for the participants in our study was 33 years, slightly lower than the average ages of 38 cited by other literature, 5 but consistent with other health research. ${ }^{16}$ More females than males attended at $\mathrm{MOHC}$, as reported by Jawaid et al. ${ }^{16}$ This finding is consistent with health seeking behaviour theories which consider that females report higher morbidity while males are prone

\begin{tabular}{|c|c|c|c|}
\hline \multirow{2}{*}{ Variables } & \multicolumn{3}{|c|}{ Percentage (Frequency) } \\
\hline & Yes & Ambivalent & No \\
\hline I think the waiting times were justified & $82.6(123)$ & $3.3(5)$ & $14.1(21)$ \\
\hline I am satisfied with the state of the hospital & $59.7(89)$ & $37.6(56)$ & $2.7(4)$ \\
\hline I am satisfied with the attending doctors & $69.1(103)$ & $26.2(39)$ & $4.7(7)$ \\
\hline I am satisfied with the quality of care given & $66.4(99)$ & $4.0(6)$ & $29.5(44)$ \\
\hline I will visit this hospital again in future & 85.9 (128) & $12.8(19)$ & $1.3(2)$ \\
\hline I will refer persons to this hospital for care & $81.9(122)$ & $14.2(21)$ & $3.4(5)$ \\
\hline
\end{tabular}

Table 6: Logistic regression analysis of factors influencing patient satisfaction

\begin{tabular}{|l|c|c|c|}
\hline & B coefficient & $95 \% \mathrm{Cl}$ & P value \\
\hline Waiting time & -0.021 & $0.96-1.00$ & 0.05 \\
\hline Time with dentist & -0.02 & $0.97-0.99$ & 0.02 \\
\hline Total time at the hospital & 0.02 & $1.00-1.03$ & 0.03 \\
\hline
\end{tabular}

to high mortality rates due to their failure to seek care early or to engage in preventive and promotive health care..$^{17,18}$

The average waiting time observed in this study is comparable to reported mean waiting times at medical facilities, ranging below the average of some studies ${ }^{5}$ and being relatively longer than other reports. ${ }^{16,19-22}$ This study, conducted in an oral and dental health care facility, did 
not reveal significantly different findings in terms of waiting time and patient perceptions from data related to medical care facilities.

The most common reason put forward by patients to account for the long waiting times is that dentists arrive late (but then spend less time in attending to them). This finding is also articulated by Feddock et al. and by Anderson et al.,23 At MOHC, as in other dental schools, dentists may be delayed due to their grappling with a multiplicity of responsibilities. These include clinical service rendering to patients, lecturing and teaching students, clinical supervision of students and research related-activities. Invariably, more time is spent per patient as compared with district health facilities, culminating in increased waiting times, extended treatment time and total time in the facility. High patient waiting times reported in the study can be attributed to large daily volumes of patients seeking oral and dental care at $\mathrm{MOHC}$, which is one of the three tertiary referral centres servicing the densely populated Gauteng province. This pattern of extended waiting times and delays is common to teaching institutions.

The organizational structure and plans for the filling of posts at $\mathrm{MOHC}$, like other public facilities, is determined largely by budgetary allocations from the Department of Health. In the recent past, the Department has experienced serious financial difficulties leading inexorably to increased vacancies at $\mathrm{MOHC}$. These have precipitated unfavourable doctor-patient ratios, which have further exacerbated long waiting times. ${ }^{5,22}$ Dental schools across South Africa report similar staff shortages, long patient volumes and waiting times. Surprisingly, few of the participants indicated a perception that dentists spend too much time in consultation with patients.

The perception of patients that dentists arrive late for clinical sessions may be an indication that standards of professionalism may be compromised. Lapses in professionalism and performance, while not objectively assessed in this study, have been reported by patients as contributing to long waiting times. ${ }^{5}$ The perception that dentists are not timeous in attending to patients is a factor that cannot be overlooked. Health personnel need continually to conduct themselves in a professional manner as their patients hold them in high esteem due to their status in the discipline Dentists may need to make greater efforts to be consistently punctual for the patients such that they are not perceived to be late or absent for service rendering. ${ }^{24}$ Alternatively, patients should be informed of how long, realistically, they may have to wait for the dentist to be available.. ${ }^{24}$

In addition, long queues for files at the administrative area was indicated as a reason for extended waiting time experienced by patients. Therefore, it is fundamental that administrative processes should be streamlined to avoid delays and improve patient flow. ${ }^{25}$ Novel engagement of patients during this time can also drastically improve the perception of waiting time and overall satisfaction. The activities to fill this time cannot be mundane and generic but need to be designed in such a way that they cater for the needs of the possible niche market of patients reporting for care. Materials such as educational videos, health talks and readable material have been used. ${ }^{26}$
However, recent literature also indicates that the use of visual art can also alter the patient's experience and behaviour pattern, ${ }^{27}$ thereby positively influencing the perception of waiting time. This finding calls for research into more activities that would, without causing stress, cater for patients who present with pain. Pain has been associated with a greater likelihood of being dissatisfied. ${ }^{28}$ Staff managing these patients should be informed of the role pain plays in perceived satisfaction and asked to proactively accommodate patients in pain in the outpatients clinics.

This study shows a positive association between satisfaction and future prospects about the services at $\mathrm{MOHC}$. The majority of patients who are satisfied with services intend revisiting the centre in the future, indicating that intention to revisit and to recommend the facility to others is a strong, significant and reliable proxy for satisfaction. ${ }^{29-31}$

\section{CONCLUSION}

Waiting time is the single most important contributor to patient satisfaction

\section{Recommendations}

The Health Manager should introduce efficient process along the entire supply chain in order to improve waiting times. Furthermore, patients should be positively engaged whilst waiting for service so as to alter their perception of waiting times. Interventions such as videos, magazines, health talks, visual art and information by oral health personnel, have proven benefits.

\section{References}

1. Hemson D,Roberts B. Batho Pele: A season of discontent. HSRC Review 2008;6(4):12-4

2. Ko YK. The relationships among waiting time, patient's satisfaction, and revisiting intention of outpatients in general hospital. Journal of Korean Academy of Nursing Administration 2010;16(3):219-28.

3. Lee MA,Yom Y-H. A comparative study of patients' and nurses' perceptions of the quality of nursing services, satisfaction and intent to revisit the hospital: A questionnaire survey. International Journal of Nursing Studies 2007;44(4):545-55.

4. Piper LE. Patients are not always rational: the leadership challenge to improve patient satisfaction scores. The Health Care Manager 2010;29(3):233-40.

5. Umar I,Oche M,Umar A. Patient waiting time in a tertiary health institution in Northern Nigeria.J. Public Health Epidemiology. 2011;3(2):78-83.

6. Al-Abri R,Al-Balushi A. Patient satisfaction survey as a tool towards quality improvement. Oman Medical Journal 2014:29(1):3

7. Hekkert KD,Cihangir S,Kleefstra SM,van den Berg B,Kool RB. Patient satisfaction revisited: a multilevel approach. Socia Science \& Medicine 2009;69(1):68-75.

8. Anderson RT,Camacho FT,Balkrishnan R. Willing to wait?: The influence of patient wait time on satisfaction with primary care. BMC Health Services Research 2007;7(1):31.

9. Linder-Pelz S. Toward a theory of patient satisfaction. Social Science \& Medicine 1982;16(5):577-82.

10. Chakraborty R, Majumdar A. Measuring consumer satisfaction in health care sector: The applicability of servqual. Researchers' World 2011;2(4):149.

11. Tucker J. Moderators of patient satisfaction. Journal of Management in Medicine 2002;16(1):44-66.

12. Wolosin R. The voice of the patient. Quality Management Health Care. 2005;14:155-64. 
13. O'Malley M,Fletcher S,Fletcher R,Earp J. Measuring patient waiting time is a practice setting: A comparison of methods. Journal of Ambulatory Care Management. 1993;6:20-7.

14. Pruyn A,A Smidts A. Effects of waiting on satisfaction with service:beyond objective measures. International Journal of Research in Marketing 1998;15(4):321-34.

15. Parasuraman A, Zeithaml VA,Berry LL. Servqual: A multipleitem scale for measuring consumer perceptions. Journal of Retailing 1988;64(1):12.

16. Jawaid M,Ahmed N,Alam SN,Rizvi BH,Razzak HA. Patients experiences and satisfaction from surgical out-patient department of a tertiary care teaching hospital. Pakistan Medical Journal 2009;25(3):439-42.

17. Möller-Leimkühler AM. Barriers to help-seeking by men: a review of sociocultural and clinical literature with particular reference to depression. Journal of Affective Disorders 2002;71(1):1-9.

18. Galdas PM,Cheater F,Marshall P. Men and health help-seeking behaviour: Literature review. Journal of Advanced Nursing 2005;49(6):616-23.

19. Bamgboye EA,Jarallah JS. Long-waiting outpatients: target audience for health education. Patient Education and Counselling 1994;23(4):49-54.

20. Net N,Sermsri S,Chompikul J. Patient satisfaction with Health services at the Out patient Department Clinic of Wangmamyen Community Hospital, Sakeao province, Thailand. Journal of Public Health and Development 2007;5(2):33-40.

21. Dos Santos L,Stewart G,Rosenberg N. "Pediatric emergency department walkouts".Pediatric Emergency Care 1994;10:76-8.

22. Thacher T. Outpatient waiting time in Jos University Teaching Hospital.Highland Medical Research Journal 2005;3(1):36-42.

23. Feddock CA,Hoellein AR,Griffith III CH, et al. Can physicians improve patient satisfaction with long waiting times? Evaluation and the Health Professions 2005;28(1):40-52.

24. Adekanye A,Adefemi S,Okuku A, et al. Patients' satisfaction with the healthcare services at a north central Nigerian tertiary hospital. Nigerian Journal of Medicine 2013;22(3):218-24.

25. Sayah A,Rogers L,Devarajan K,Kingsley-Rocker L,Lobon LF. Minimizing ED waiting times and improving patient flow and experience of care. Emergency Medicine International 2014; 981472.doi:10.1155/2014/981472. Epub 2014 Apr 14.

26. Ajayi IO. Patients' waiting time at an outpatient clinic in Nigeria-can it be put to better use? Patient Education and Counselling 2002;47(2):121-6.

27. Nanda U,Chanaud C, Nelson M, et al. Impact of visual art on patient behavior in the emergency department waiting room. Journal of Emergency Medicine 2012;43(1):172-81.

28. De Brun C,Howell F,Bedford D,Corcoran R,Kelly A. Outpatient experiences in acute hospitals. Irish Journal of Medical Science 2002;171(2):89-93.

29. Lexshimi R,Zaleha M,Shamsul A,Suriawati G. Patient satisfaction on waiting time and duration of consultation at orthopaedic clinic, Universiti Kebangsaan Malaysia Medical Centre. Medicine \& Health 2009;4(1):35-46.

30. Park Y-D,Jang E-J. Factors of the quality of dental care service upon intention to revisit. Journal of Korean Academy of Dental Technology 2011;33(4):441-52.

31. Thompson AG,Sunol R. Expectations as determinants of patient satisfaction: concepts, theory and evidence. International Journal for Quality in Health Care 1995;7(2):127-41. 